\title{
Diseño de una guía para la administración del riesgo de operación con tirador escogido de plataforma
}

\section{CT. Hugo Valderrama Vivas \\ CT. Luis Miguel Díaz Ríos}

\section{Descripción}

Esta investigación es la primera apreciación que se hace de manera lógica y organizada de los pasos que se deben seguir durante la configuración de una aeronave de la Fuerza Aérea, para realizar operaciones especiales con tiradores escogidos de plataforma. Se presentan los resultados de la aplicación de una estrategia específica en una aeronave prototipo: el avión King Air C-90, para perfeccionar el alistamiento, operación y evaluación a través de una guía con un enfoque operacional de seguridad aérea, hacia el área de la prevención de accidentes, dirigida a advertir acerca de inconvenientes, fallas o problemas que se pueden presentar con base en la experiencia de la operación del GRUPO AÉREO DEL CARIBE, la cual puede ser aplicada en el resto de aeronaves de ala fija y rotatoria de la Fuerza Aérea Colombiana.

\section{Objetivos}

\section{Objetivo general}

Diseñar una guía para la administración del riesgo de operación con tirador escogido de plataforma aérea, que permita aumentar y mantener la seguridad aérea y el mejor desarrollo de operaciones aéreas.

\section{Objetivos específicos}

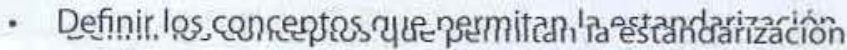
de un lenguaje técnico para el personal involucrado en el desarrollo de operaciones con tiradores escogidos de plataforma aérea.

- Determinar los procedimientos de operación con equipo de tiradores escogidos de plataforma aérea y sus respectivas capacidades a través de la descripción de los elementos que se deben aplicar durante el desarrollo de la operación.

- Desarrollar los modelos de manejo de riesgos durante el desarrollo de las operaciones, acompañado de la elaboración de los flujogramas, para la administración de los riesgos en cada fase de la operación de las aeronaves con equipo de tiradores escogidos de plataforma aérea.

- Diseñar la guía con los procedimientos, listas de verificación conjuntas para la tripulación, tiradores y comandantes, que permitan los registros de datos para la seguridad de vuelo durante las operaciones con el equipo de tiradores escogidos de plataforma aérea.

\section{Metodología}

Es de carácter documental por la fuente y el análisis de la información, ya que el trabajo está enfocado hacia la definición y conceptualización de los términos que se utilizaron durante la creación de las listas de chequeo que se diseñarôn, 'ya quíe no no se cuenta con estos procedimientos por ser una operación nueva y $\sin$ antecedentes. $Y$ descriptiva, en 
las siguientes fases ya que se toma como modelo y prototipo de configuración para el desarrollo el avión King Air C-90; con base en éste se describieron las partes requeridas y se determinaron los procedimientos que se deben aplicar de acuerdo con la doctrina aérea para finalizar con el desarrollo de las listas y modelos en forma de flujogramas de manera didáctica, que permitieron la valoración en tiempo real, así como la evaluación y retroalimentación requerida para el mejoramiento de los procedimientos guía en esta operación especifica y con el fin de que sean homologadas en las aeronaves de la Fuerza Aérea que efectúan esta misión típica como el AC-47T Fantasma, UH-60 Black Hawk, UH-1P HUEY II y el B-212, teniendo en cuenta que no existe ningún antecedente escrito o bibliografía que sirva de punto de partida para este tipo de operación en una aeronave.

\section{Resultados}

Durante el diseño de la guía para la administración del riesgo de operación con tirador escogido de plataforma aérea se demostró la necesidad de enmarcar cada misión dentro de los pasos fundamentales que se deben aplicar en la operación teniendo en cuenta que, como Fuerza Aérea y pioneros en este tipo de misión, se tiene la obligación de liderar y marcar el nivel cultural requerido para la defensa de la nación y la aplicación de la Fuerza por medio de las armas con soporte legal para no caer en errores y aportar reglas para una doctrina táctica que aumente los niveles de seguridad aérea, con principios inmutables y fijos que fortalezcan el poder aéreo nacional y la vigencia de las reglas propias del estado.

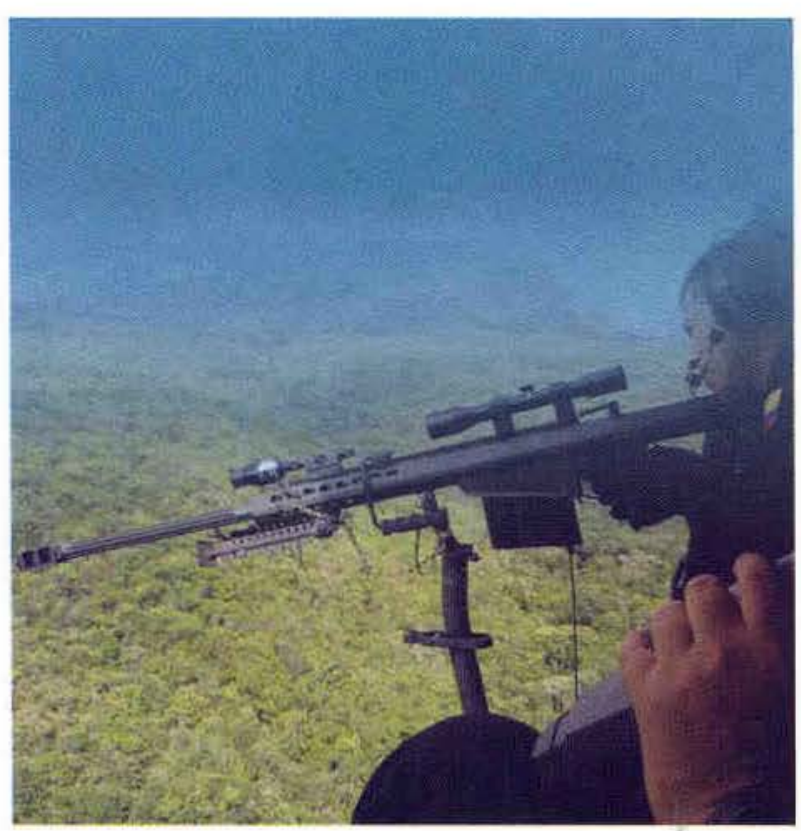

Igualmente, se caracterizó un flujograma de administración de operación TEPLA, que permitirá supervisar los procedimientos y políticas fijadas al respecto a lo largo de la guía para administrar la operación de manera didáctica como un recurso de diseño y aprendizaje de cada operación. Finalmente, se ajustó a los programas de prevención y promoción de seguridad aérea durante las operaciones especiales.

\section{Bibliografia}

CARTILLA DOCTRINA AEREA. Operaciones aéreas segunda edición 2004

Normas y procedimientos para la operación de los tiradores escogidos de plataforma aérea y superficie de la FAC. COFAC $08-30-3492005$

CODY, Frank J y DISE, John H. Jr. Manual of Educational Risk Management. Detroit, Michigan: Educational Risk, inc, 1991. 984 pages.

\section{CONSTITUGIÓN POLITICA DE COLOMBIA art. 101,217.}

CHENG, Rita Hartung y YAHR, Robert B. "Risk Management Practices and Accounting Requirements." School Business Affairs 55,10 (October 1989):20-27, EJ 397766 .

DUNKLEE, Dennis R. y SHOOPRobert J. A Primer for School Risk Management: Creating and Maintaining District and Site-Based Liability Prevention Programs. Boston: Alyn and Bacon, 1993. 209 pages.

GAUSTAD, Joan C. Risk Management: How School Districts Can Identify Risks, Reduce Losses, and Conserve Funds. Eugene, Oregon: Oregon School Study Council, University of Oregon, September 1993. OSSC Bulletin Series, 40 pages.

JOHNSON, Brad. "Walking the Risk Tightrope: Balancing Risk Finance Options:" School Business Affairs 58, 6 (June 1992): 4.9.

JOHNSTON, James B. "Transfer of Risk: Plan Now or Pay Later." School Business Affairs 59, 6 (June 1993): 10-13. EJ 465290.

MANUAL DE OPERACIONES AÉREAS (O-MAOPA) FAC 3-62 RESERVADO

MINOR, Jacqueline $K$ y MINOR, Vern B. Risk Management in Schools: A Guide to Minimizing Liability. Newbury Park, California: Corwin Press, 1991.82 pages.

MORLEY, John. "Community Use of Schools: A Risk Management Approach." School Business Affairs 56, 6 (June 1990): 20-24. EJ 411638

PENSAMIENTOS SOBRE LA HUMANIDAD. Luis Alberto Escobar, Panamericana (2003)

RANDAL, L. Nathan. "Risk Management" In Principles of School Business Management, edited by R. Craig Wood. Reston, Virginia: Association of School Business Officials International, 1986. 675 pages. ED 282294.

REGLAMENTO DE LA HAYA. Sobre leyes y costumbres de la guerra 1907, anexo IV, art.2, protocolo l, art.23, 41 Y 46, Convenio, de Ginebra1980. 